\title{
12. Recurrence Risk for the Second 21-Trisomic Children
}

\author{
By Hiroko FuJita, *) Yasuko Hirano,*) Tadashi Sugawa,**) \\ Sachio OGITA,**) Naoki Nomoto,***) \\ and Ryuichi TsukINo****) \\ (Communicated by Sajiro Makıno, M. J. A., Feb. 13, 1978)
}

The recurrence risk of non-disjunction in a family is noted as one of important medical problems in relation to the etiology of Down's syndrome. The present report deals with the risk of producing a second child with 21-trisomy.

Material and methods. An attempt was made to collect, in a large scale, Down's syndrome patients so far obtained in laboratories in the Kansai district during the last decade. Data for this study were derived from 750 Down's syndrome cases who received cytological examinations in the major 12 laboratories of this district. They were born during a period from 1966 to 1975. They were categorised into 5 groups according to their karyotypes as shown in Table I. The propotional picture of the karyotype is in accordance with that of the previous reports.

Table I. Karyotypic distribution of patients with Down's syndrome and recurrent family

\begin{tabular}{lrcccc}
\hline \multirow{2}{*}{ Karyotype } & \multirow{2}{*}{ Numbers (Inheritance) } & Incidence & \multicolumn{2}{c}{ Recurrent family } \\
\cline { 3 - 5 } & & & $\%$ & Observed & Expected \\
\hline $47,+21$ & 690 & $(9)$ & 92.3 & 6 & $0.69 *$ \\
$46,($ Dq21q) & 23 & $(3)$ & 1.6 & 1 & $>3$ \\
$46,($ Gq21q) & 14 & 2.7 & 0 & $>1$ \\
$46, / 47,+21$ & 20 & & 0.3 & 0 & \\
Others & 3 & & 100.0 & & \\
\hline Total & 750 & &
\end{tabular}

Partial data were published by Matsunaga and Fujita (1977).

* The incidence is calculated as $0.1 \%$.

Results. In 690 cases with $47,+21$ under consideration, there were 5 sibships with two affected children and one sibship with three affected ones. There are some items in them common to each other as below (Table II) :

\footnotetext{
*) Department of Child Health, Osaka City University.

**) Department of Obstetrics and Gynecology, Osaka City University.

***) Department of Pediatrics, Kyoto City Hospital.

****) Department of Pediatrics, Wakayama Medical College.
} 
1) On the whole, the birth rank of the affected children in a sibship was the first, the second and the 3rd birth in a successive series.

2) Maternal age at the birth of the 1st child ranged from 23 to 29 years, the mean age being 25.5 years. This is identical to the mean maternal age of the first child in the general population, but younger than that of the 289 first child with 21-trisomy of this study (Table III).

3) Karyotypes of all parents so far examined were shown as normal according to the routin leucocyte analysis.

Table II. Sibships with more than two Down's syndrome

\begin{tabular}{|c|c|c|c|c|c|c|}
\hline & \multirow{2}{*}{ Case } & \multirow{2}{*}{ Birth rank } & \multirow{2}{*}{ Birth date } & \multirow{2}{*}{$\begin{array}{l}\text { Maternal } \\
\text { age }\end{array}$} & \multicolumn{2}{|c|}{ Karyotype } \\
\hline & & & & & Father & Mother \\
\hline \multirow{13}{*}{ 21-trisomy } & \multirow{2}{*}{1} & I & 1967 & 27 & \multirow{2}{*}{ Normal } & \multirow{2}{*}{ Normal } \\
\hline & & II & 1975 & 35 & & \\
\hline & \multirow{2}{*}{2} & $\mathrm{I}$ & 1974 & 24 & \multirow{2}{*}{ Normal } & \multirow{2}{*}{ Normal } \\
\hline & & II & 1975 & 25 & & \\
\hline & \multirow{3}{*}{3} & I & 1972 & 25 & \multirow{3}{*}{ Normal } & \multirow{3}{*}{ Normal } \\
\hline & & II & 1974 & 26 & & \\
\hline & & III & 1974 & 27 & & \\
\hline & \multirow{2}{*}{4} & I & 1969 & 27 & \multirow{2}{*}{ Normal } & \multirow{2}{*}{ Normal } \\
\hline & & II & 1973 & 31 & & \\
\hline & \multirow{2}{*}{5} & I & 1966 & 25 & \multirow{2}{*}{$\begin{array}{l}\text { Not } \\
\text { examined }\end{array}$} & \multirow{2}{*}{ Normal } \\
\hline & & II & 1971 & 29 & & \\
\hline & \multirow{2}{*}{6} & $\mathrm{I}$ & 1958 & 24 & \multirow{2}{*}{ Normal } & \multirow{2}{*}{ Normal } \\
\hline & & II & 1968 & 34 & & \\
\hline \multirow{2}{*}{ Dq21q } & \multirow{2}{*}{7} & I & 1871 & 23 & \multirow{2}{*}{ Carrier } & \multirow{2}{*}{ Normal } \\
\hline & & II & 1973 & 25 & & \\
\hline \multirow{2}{*}{ Gq21q } & \multirow{2}{*}{8} & I & 1964 & 25 & \multirow{2}{*}{ Carrier } & \multirow{2}{*}{ Normal } \\
\hline & & II & 1966 & 27 & & \\
\hline
\end{tabular}

Referring to the data given in Tables II and III, the recurrence risk for the parents who had the 1 st born child showing $47,+21$ can be estimated as about $2 \%(6 / 289)$. In addition to the birth rank, when their maternal ages are limited to 29 years and below, the recurrence risk can be obtained as about $2.5 \%(6 / 221)$.

For the study of the translocation, 23 patients with the Dq21q type and 14 with the Gq21q type were subjected in this survey. In the 23 Dq21q patients, 9 were of familial inheritance, in which 7 mothers and 2 fathers were translocation carriers. Out of the remaining 
Table III. Mean maternal age (MMA) in relation to birth rank

\begin{tabular}{|c|c|c|c|c|}
\hline \multirow{2}{*}{ Birth rank } & \multicolumn{2}{|c|}{ Recurrence family } & \multicolumn{2}{|c|}{ 21-trisomy group } \\
\hline & MMA & No. of cases & MMA & No. of cases \\
\hline I & 25.5 & 6 & 27.0 & $289 \begin{array}{ll}-29 * & 30-* * * \\
(221, & 68)\end{array}$ \\
\hline II & 30.5 & 6 & 29.4 & $260(149,111)$ \\
\hline III & 27.0 & 1 & 33.6 & $71(14,57)$ \\
\hline IV & & & & $22(1,21)$ \\
\hline $\mathrm{V}$ & & & & $7(0,7)$ \\
\hline VI & & & & $1(0$ \\
\hline Unknown & & & & 40 \\
\hline Total & & & & 690 \\
\hline
\end{tabular}

* 29 years and below: Maternal age at the birth of the affected child.

** 30 years and above: Maternal age at the birth of the affected child.

patients, 9 parents had a normal karyotype, the translocation of the patients being sporadic, and 5 families failed to be examined. The Gq21q type included 3 inherited patients through 2 mothers and one father, and 7 sporadic. Four families remained without examination. Amongst these translocation cases, the recurrence was found in one family, each for the Dq21q type and the Gq21q type. There is a steady decrease in the occurrence of the familial Down's syndrome with translocation karyotypes, as cytogenetic counseling has been in progress to a considerable extent around the Kansai district.

Remarks. Tonomura et al. (1974) found five sibships with two 21-trisomic children among 818 families in which the first born child was 21-trisomic. Since the parents of the above first cases were cytogenetically normal, no mosaicism having been detected in both lymphocytes and skin fibroblasts, they estimated, on the basis of numbers in the second child, the recurrence risk as $5 / 512=1 / 98$. Uchida (1970) calculated the risk of producing a second trisomic child as one to 57 in Manitoba, Canada, based on the fact that four reproducing families were found in a total of 229 postproband pregnancies of the trisomic families. Hamerton et al. (1961) mentioned that, if the parents were cytologically normal, the risk of recurrence after the birth of one child with 21-trisomy was about $1 \%$, regardless of maternal age. The total incidence of Down's syndrome in the general population of Japan in a period from 1970 to 1975 was calculated as $0.101 \%$ (Matsunaga and Fujita, 1977). If the recurrence would occur by chance, the second affected child may be born with the ratio of one to a thousand postproband births. Our data on the families with two or more trisomic children seem to be unfavorable for the view that the cause of recurrent non-disjunction is dependent merely upon the maternal age. Another mechanism involving the interference between 
pairing and disjunction other than the maternal age may become possible in terms of cytological pictures including the short arms with satelites of the D and G group chromosome, or of genetic features known in certain organisms other than man. Tajirova and Klein (1971) expressed a view that the tendency to non-disjunction is connected with an increase in the number of joined chromosomes during cell division. Similarly, Ballantyne et al. (1977) is of opinion that the 21 chromosome with deleted short arm would tend to produce irregularities in the normal disjunction of this chromosomes and its homologus.

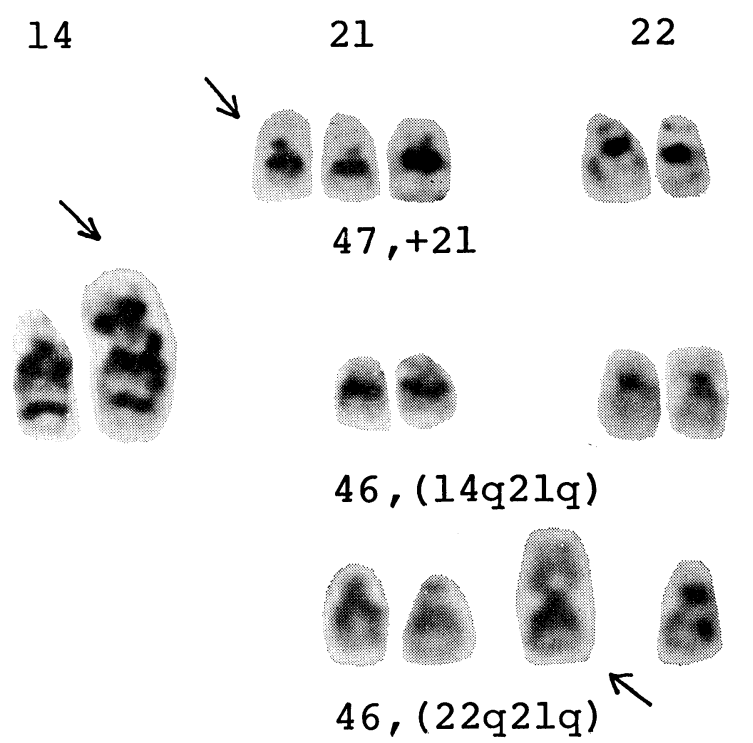

Fig. 1. Partial G-banded karyotypes of Down's syndrome. Arrows indicate additional 21-chromosome in each.

Etiological investigation of non-disjunction is a subject of prime importance for causal analysis of the chromosome abnormality in relation to the congenital disorders of man.

Acknowledgment. Our cordial thanks are due to Emeritus Professor Sajiro Makino, M. J. A., for invaluable advice. Thanks are extended also to many doctors and collaborators who attached to the Hyogo Prefectural Tsukaguchi Hospital, Aizenbashi Hospital, Osaka Red Cross Hospital, Tenriyorozu Hospital, Hyogo Prefectural Children's Hospital, Osaka Children's Medical Center, Nara Medical College and Hyogo Medical College, for providing invaluable information necessary for the present study. 


\section{References}

Ballantyne, G. H., Parslow, M. I., Veale, A. M. O., and Pullon, D. H. H.: J. Med. Genet., 14, 147 (1977).

Hamerton, J. L., Briggs, S. M., Gianelli, F., and Carter, C. O.: Lanset 2, 788 (1961).

Tajirova, O., and Klein, D.: J. Gènèt hum., 19, 1 (1971).

Tonomura, A., Aoki, H., and Kishi, K.: Jap. J. Hum. Genet., 20, 73 (1975).

Uchida, I. A.: Ann. N. Y. Acad. Sci., 171, 361 (1970).

Matsunaga, E., and Fujita, H.: Hum. Genet., 37, 221 (1977). 